\title{
The segmented aperture interferometric nulling testbed (SAINT) III: control systems analysis and preliminary results
}

Brian A. Hicks, Keith Jahoda, Peter Petrone, Teresa Sheets, Patricia Boyd

Brian A. Hicks, Keith Jahoda, Peter Petrone, Teresa Sheets, Patricia Boyd, "The segmented aperture interferometric nulling testbed (SAINT) III: control systems analysis and preliminary results," Proc. SPIE 10698, Space Telescopes and Instrumentation 2018: Optical, Infrared, and Millimeter Wave, 106986M (12 July 2018); doi: 10.1117/12.2318756

Event: SPIE Astronomical Telescopes + Instrumentation, 2018, Austin, Texas, United States 


\title{
The Segmented Aperture Interferometric Nulling Testbed (SAINT) III: control systems analysis and preliminary results
}

\author{
Brian A. Hicks ${ }^{*} a, b$, Keith Jahoda ${ }^{b}$, Peter Petrone III ${ }^{c, b}$, Teresa Sheets ${ }^{b}$, Padi Boyd ${ }^{b}$ \\ ${ }^{a}$ University of Maryland, College Park, MD, USA \\ ${ }^{b}$ NASA Goddard Space Flight Center, Greenbelt, MD, USA \\ ${ }^{c}$ Sigma Space Corporation, Lanham, MD, USA
}

\begin{abstract}
This work presents a detailed current performance analysis for the telescope, pointing, and coronagraph component subsystems of the Segmented Aperture Interferometric Nulling Testbed (SAINT). The project pairs an active segmented mirror with the Visible Nulling Coronagraph (VNC) towards demonstrating capabilities for the future space observatories needed to directly detect and characterize Earth-sized worlds around nearby stars. We describe approaches to optimize subsystem wavefront sensing and control parameters, summarizing relevant scaling relations between these parameters, residual errors, and observed contrast measurements. Preliminary results from diagnostic testing under various control states are presented along with intermediate contrast measurements towards demonstrating the full system.
\end{abstract}

Keywords: Exoplanets, high-contrast imaging, nulling interferometry, wavefront sensing and control, space telescopes, segmented mirrors

\section{PROJECT SCOPE}

Identifying and characterizing potentially habitable planets around nearby stars are motivating science goals for future space telescopes. The telescopes will need to be large to address exoplanet direct imaging resolution and photometric flux constraints and may likely build upon hexagonal array segmented mirror infrastructure in order to fit inside of limited launch fairing volumes. Sensitivity to any existing Earth-like planets orbiting the many hundreds of nearby stars may be achieved by pairing an adequately-sized telescope with a coronagraphic starlight suppression system. Controlling optical elements to find and hold an alignment solution at unprecedented levels of precision in the presence of dynamic perturbations presents a formidable challenge for increasingly complex laboratory experiments striving towards higher fidelity demonstrations.

In support of NASA's Exoplanet Exploration Program and the Technology Demonstration for Exoplanet Missions (TDEM $)^{1}$ component of NASA's Strategic Astrophysics Technology (SAT) program, the Visible Nulling Coronagraph (VNC) at NASA's Goddard Space Flight Center is being coupled with an actively-controlled macro scale segmented primary mirror via a fine pointing system (FPS) to form the Segmented Aperture Interferometric Nulling Testbed (SAINT). ${ }^{2,3}$ SAINT is being developed as an end-to-end coronagraphic system to demonstrate high-contrast imaging in the presence of complex diffraction and environmental instabilities with traceability to future large space telescopes having coronagraphic high-contrast imaging capability. This TDEM-13 program's final Milestone (MS 3) objective comprises holding a contrast of $10^{-8}$ at an inner working angle (IWA) of $4 \lambda / D$ within a bandpass of $\Delta \lambda=20 \mathrm{~nm}$ centered near $633 \mathrm{~nm}$ for 1,000 seconds on three separate occasions, with a goal of $10^{-9}$ at an IWA of $3 \lambda / D$ over a $\Delta \lambda=40 \mathrm{~nm}$ bandpass. Intermediate Milestone goals include reproducing or exceeding earlier TDEM ${ }^{4,5}$ narrowband performance (MS 1.5), and achieving for the first time a broadband high-contrast demonstration with the VNC alone (MS 2.5). ${ }^{6-8}$ Satisfying these TDEM milestone objectives are the next steps in establishing the VNC as a viable option for a future large space telescope requiring capability for directly detecting and characterizing Earth-like exoplanets.

In this paper we present single frame contrast and stability measurements for the VNC, as well as baseline measurements of end-to-end SAINT imaging performance and image centroid jitter analysis. Performance requirements are presented for the end-to-end system including fine alignment of the segmented primary (M1) and closed-loop FPS control.

\footnotetext{
*bahicksmail@gmail.com
} 


\section{SYSTEM OVERVIEW}

The SAINT system pairs an actively-controlled segmented telescope operating in an enclosure within an ambient laboratory environment with the VNC, which operates inside a vacuum chamber. The chamber provides an isolated environment for demonstrations at atmospheric pressure, at reduced pressure similar to a stratospheric balloon environment, or at high vacuum to emulate system operation in a space-like environment. The vacuum chamber rests atop a passively stabilized air table that supports the full system. Vacuum feedthroughs consisting of electrical cables and water chiller lines to cool the high-contrast focal plane detector pass through multiple tank bulkheads. Fig. 1 shows a raytrace of the end-to-end optical system. The telescope optics form one of two selectable source inputs for the VNC, the other being a circular aperture with Gaussian intensity profile for intermediate milestone narrowband and broadband demonstrations of the $\mathrm{VNC}$ alone.
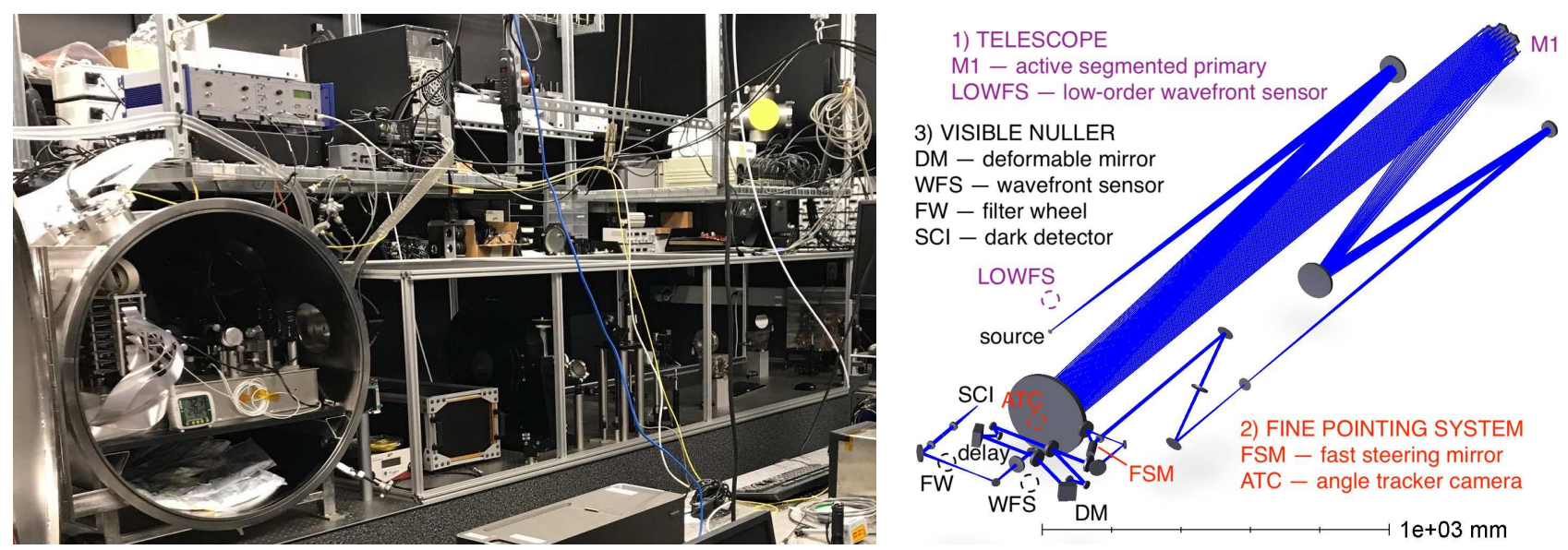

Figure 1. A photo of the SAINT system showing all but two control computers and a raytrace showing the end-to-end system path. The approximate locations are labeled for each subsystem's sensing and control components. In addition to the labeled components, each arm of the VNC has a shutter that can be closed to calibrate imaging quality, measure source brightness fluctuations, as well as to measure and balance intensities on the high contrast detector in the dark, symmetric output of the nuller.

The computer (CPU) interface and software control development for the full system is presently distributed between three computers, all running the same Linux OS. The first CPU interfaces the SAINT M1 low-order wavefront sensor (LOWFS) detector \& wavefront control (WFC) in the form of rigid body piston/tip/tilt (PTT) actuation of segments via 3-axis piezoelectric transducer ("piezo" or PZT) mounts. The second CPU interfaces the FPS fast steering mirror (FSM) amplifiers for the tip-tilt PZT stage and readout for strain gauge sensors, providing closed-loop displacement feedback for each axis, as well as an angle tracker camera (ATC) to close the loop on stabilizing pointing jitter. The third CPU commands and reads out components of the VNC including the DM, shutters, delay stage, the "dark" channel high-contrast science detector (SCI), and the high-order "bright" channel WFS.

\subsection{Filtered source diffraction measurements}

Source brightness, system transmission, diffraction, aberrations, coatings and surface errors, magnification, as well as detector dynamic range and noise all play a role in determining control rates and the number of exposures required to measure an arbitrary scattered light residual with arbitrary confidence for a given detector over a given bandpass. Here we present data characterizing the imaging performance of the VNC expected using color filters selected for the intermediate narrowband and broadband Milestones (MS), MS 1.5 and MS 2.5, respectively, that will be traceable to the SAINT final end-to-end MS 3 performance. The central wavelength, $\lambda$, and full-width at half-maximum, FWHM, was previously determined for each filter from spectrophotometer transmission measurements. ${ }^{9}$

Figures 2 and 3 show cross-sections through the point spread function (PSF) observed by the VNC dark detector under faint source and high background and bright source low background conditions, respectively, 
using each of the two intermediate and final Milestone filters. The ratio of core to sidelobe intensities can be measured and used to increase the maximum contrast in a single frame. To illustrate this, the exposure times were set for the Milestone 1.5 filter such that the core does not saturate in either the faint or bright source conditions.

Table 1. VNC/SAINT imaging bandpass characteristics

\begin{tabular}{lcccccc} 
filter & $\lambda(\mathrm{nm})$ & FWHM $(\mathrm{nm})$ & $\Delta \lambda / \lambda(\%)$ & $\lambda^{2} / \Delta \lambda$ (waves) & $\tau(\mathrm{ms})$ & rate (fps) \\
\hline \hline MS 1.5 & 632.8 & 1.26 & 0.20 & 502 & 2.00 & 45 \\
MS 2.5 & 654.7 & 31.7 & 4.84 & 21 & 0.25 & 50 \\
MS 3 & 649.2 & 17.5 & 2.70 & 37 & 0.25 & 50 \\
\hline
\end{tabular}

In final contrast demonstrations the VNC will be operated in vacuum using in total two single mode fiber patch cables and a single mode fiber feedthrough that all have cutoff wavelengths in the range of $500-600 \mathrm{~nm}$. The bright source exposure times, $\tau$, and frame rates presented in Tab. 1 were measured without a vacuum fiber feedthrough and additional single mode fiber patch to the VNC input. Assuming a modest $60 \%$ coupling efficiency into a vacuum feedthrough and fiber patch, peak intensities under otherwise identical conditions would fall below saturation and the Milestone (MS) 1.5 peak intensity would be approximately 2.7 times lower than what is observed in Fig. 3.
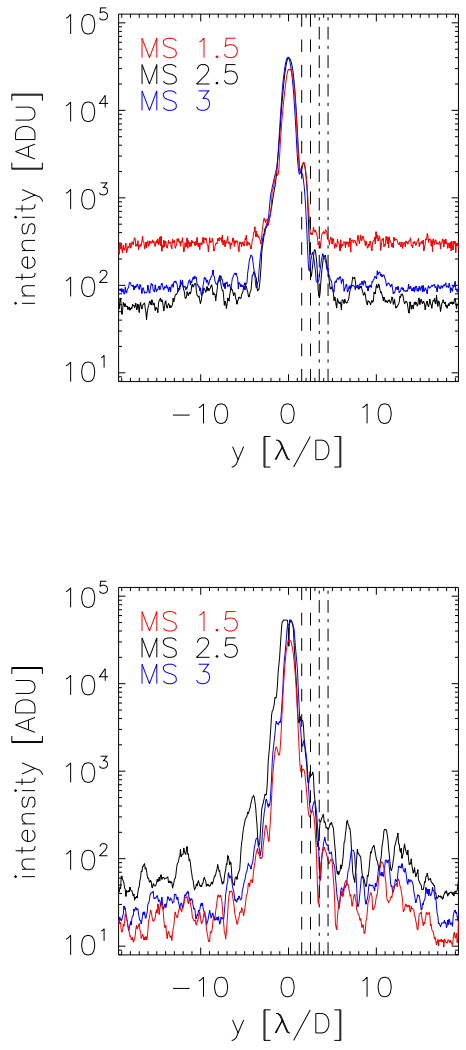
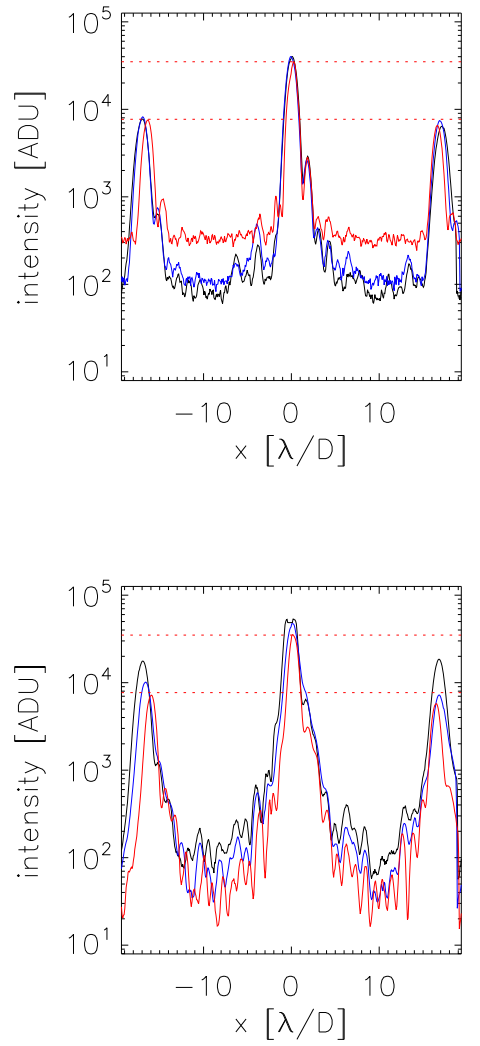

Figure 2. PSF cross-sections observed with the VNC dark detector under faint source and ambient dim lab background conditions. As expected, off-axis pixel intensities are observed to increase with longer integrations of dark current and background. The dashed lines correspond to the contrast measurement critical boundaries for MS 1.5 and 2.5, and the dashed-dotted lines correspond to the MS 3 critical boundaries.

Figure 3. The same as Fig. 2, but under bright source, dark chamber, low background, demonstration-like conditions using exposure times listed in Tab. 1. The MS 2.5 and MS 3 PSF cores are saturated.

For each bandpass, the measured peak intensity is between one and two orders of magnitude greater than the intensity measured in the off-axis range critical to the Milestone demonstrations. Under the condition of destructive interference, the exposure times can be increased to improve the scattered light signal residual, but only to the limit where perturbations above the resultant maximum control sampling frequency do not exceed the RMS wavefront error requirement. 


\section{NULLER AND NULLER SOURCE STABILITY}

The Visible Nulling Coronagraph (VNC) is described in varying levels of detail in several previous works. ${ }^{4,6,8}$ Here we present stability measurements of the VNC in its heritage configuration, i.e. with a single mode fiber source emanating from a fixed point, mounted on the nuller breadboard inside of closed vacuum chamber at atmospheric pressure and room temperature. Results from multiple measurements are presented, starting with a time series measurement of centroid motion and intensity fluctuations with a shutter in the deformable mirror arm of the interferometer closed, followed by analysis of nulling data, both collected with control optics powered, but inactive.
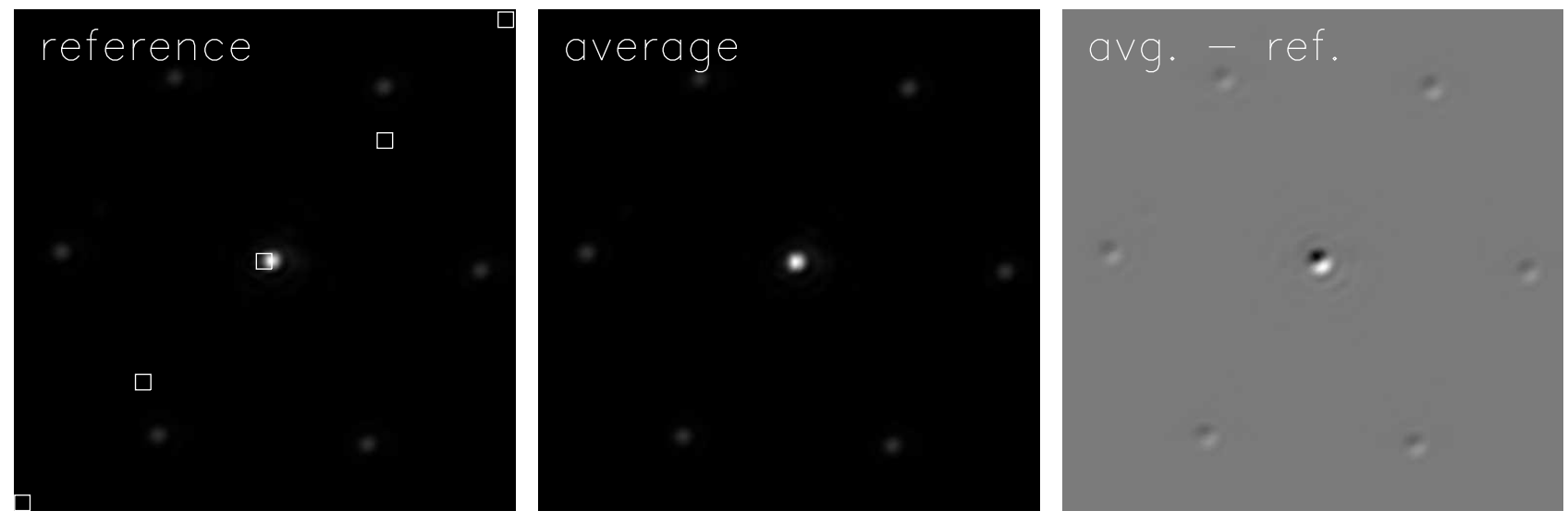

Figure 4 . Representative $512 \times 512$ pixel frames for the time series and power spectra presented in figures 5, 7, and 6 . The five boxes and their centers contain the pixels used in central single pixel and multiple pixel average time series and power spectra. The core and sidelobe diameters and separations are characteristic of the deformable mirror 7-ring hexagonal array and the corresponding Lyot mask's pitch and hole diameters. ${ }^{5,10}$ The reference frame is the first frame acquired in the series. The sidelobe centers are spaced $60^{\circ}$ at a radial distance of $16.166 \lambda / D$ from the core center, giving a plate scale of $0.07573 \lambda / D$ per pixel.

A total of 1000 frames were saved at an average rate of 18.52 per second. Representative frames of the reference arm only time series are shown in Fig. 4. The time series and power spectra of the data set's full frame measured intensity statistics are presented in Fig. 5 . The power spectrum of the frame maximum appears to $1 / \mathrm{f}$ above a need that occurs at less than 1Hz. The PSF core spans several pixels, and Fig. 4 reveals drift across several pixels over several tens of seconds. Experimentation at different magnifications under otherwise identical conditions might reveal whether the observed spectrum can be attributed to aliasing at pixel active area scales.

Time series and power spectra for the VNC single arm data set are presented in Fig. 5 for five equally spaced individual pixels, as well as approximately $1 \lambda / D$ square box averages around these pixels. The locations and boxes as shown to scale in Fig. 4 spanning a diagonal across the PSF, including a location near the core. The
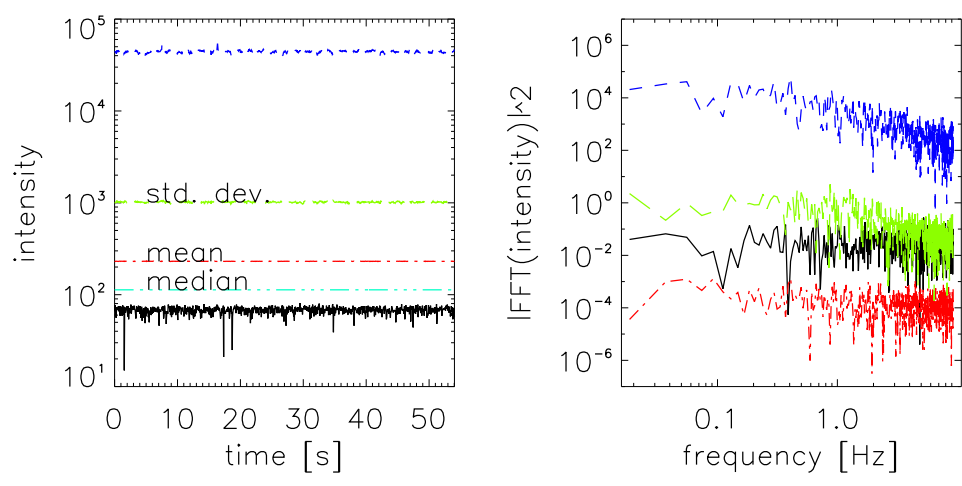

Figure 5. Fundamental image properties including the minimum, maximum, mean, median, and standard deviation for each frame of single arm VNC data under bright source and low background conditions. The mean and RMS variation of the maximum pixel value over the field of view are 44159.8 and 1471.25 ADU, respectively. The mean and RMS values of the frame mean time series is 230.910 and 0.437117 ADU, respectively. 

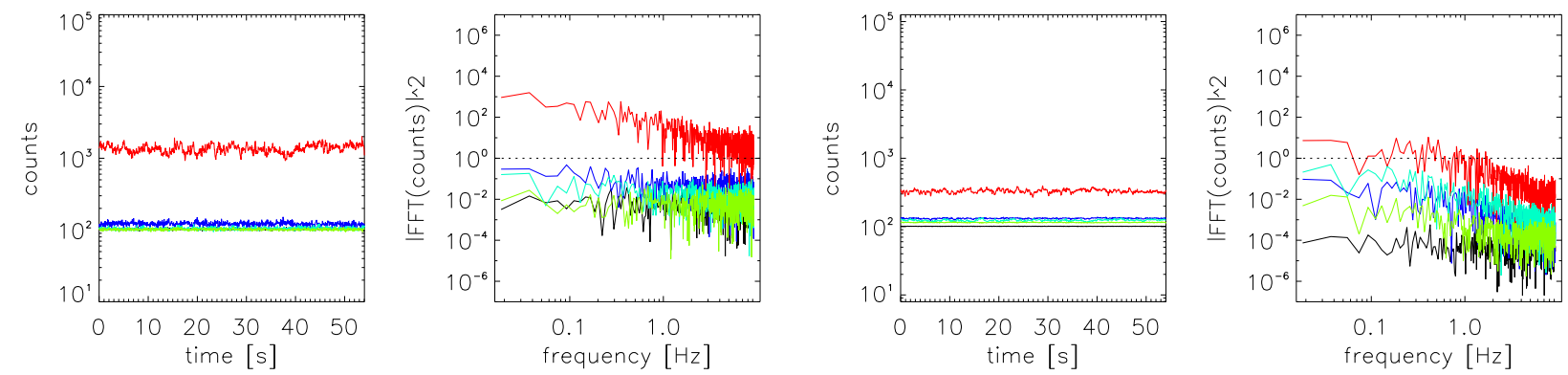

Figure 6. Time series and power spectra for the VNC delay reference arm measured under bright source and low background conditions at single pixel (left) and box-averaged (right) detector locations identified in Fig. 5.

measured signal residual in the wings of the PSF approaching the off-axis radial separation of the side lobes and in the corners of the frames is less than $10 \sigma$ above the detector bias signal. The intensity power spectra are white for individual pixels at all locations except near the core where the spectrum is pink, and pink at all locations for the box averages.

Finally, we analyze plots of centroid jitter time series and power spectra for the VNC single arm data set at hand. Jitter plots are presented in Fig. 7 as absolute motion, $\Delta$ pixel, in the focal plane relative to the mean frame centroid as a reference (top), and as instantaneous frame by frame motion (bottom). The left plots are made from the raw frames, and the right plots are the same data reanalyzed following numerically shifting each frame by fractional pixel centroid offsets in both the absolute and instantaneous treatments to emulate effects of residual errors following pointing correction. Orthogonal jitter components are plotted as dashed and dashed-dotted lines in blue and red, respectively, and the magnitude of the jitter is plotted as a solid black line. The RMS jitter calculated from the raw and shifted data residual relative to the average frame is 0.798 and 0.168 pixels, respectively. For the instantaneous treatment, the same values are 0.131 and 0.033 . The absolute treatment reduces the RMS centroid jitter by 4.75 times, whereas the instantaneous treatment reduces the error by 3.97 times, and ultimately achieves an RMS error that less than $20 \%$ of the RMS error achieved referencing
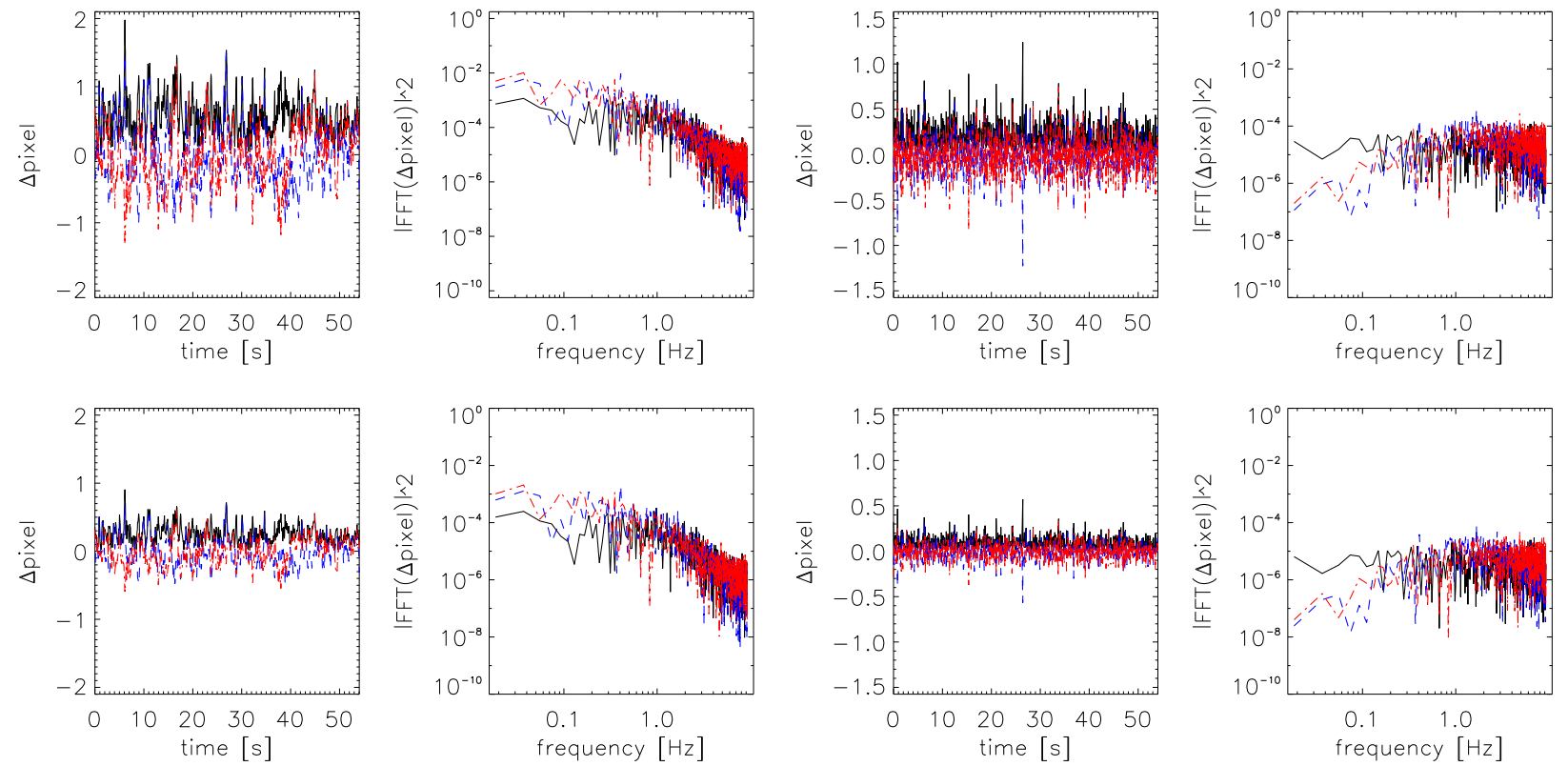

Figure 7. Single arm VNC centroid jitter time series and power spectra plotted for both $\mathrm{x}$ and $\mathrm{y}$ directions, as well as magnitude relative to the mean centroid location (top), and previous frame instantaneous centroid motion (bottom). 

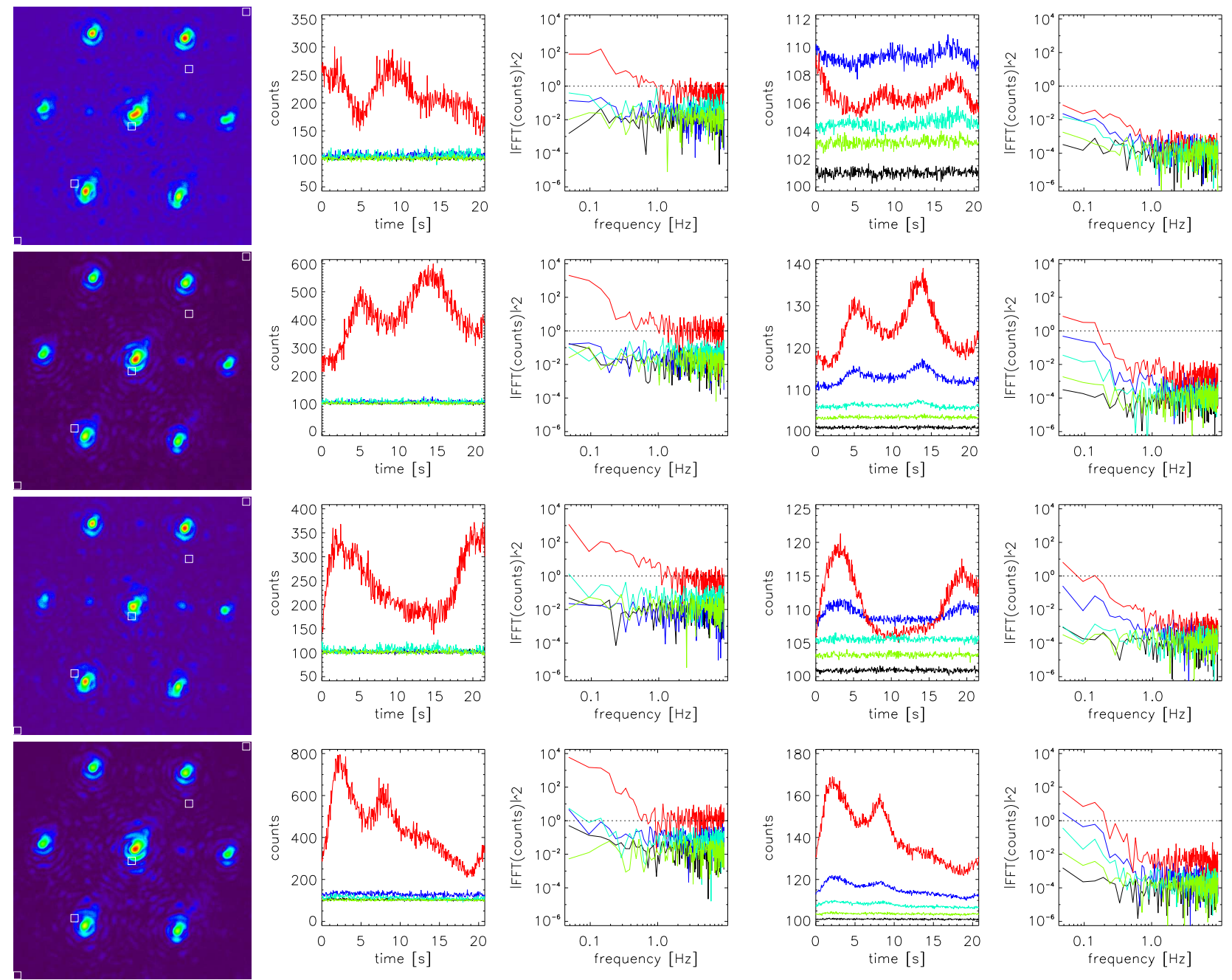

Figure 8. Logarithmically stretched interferometric null first frames (left) next to their corresponding time series and power spectra for individual pixels centered in each of the five indicated box locations (center), and time series and power spectra averaged over all pixels in each box (right).

relative to the average of all frames.

Next, in Fig. 8, we present data similar to what is shown in Fig. 6, but for the case of operating near a destructive interferometric null in four data collection events (DCEs) consisting of 400 consecutively acquired frames. Time series are measured at the same five image locations in each of the DCEs. Similar to the single arm measurements, both multipixel averages as well as single pixel intensities are plotted for each DCE.

The plots in Fig. 8 convey both the advantage if not the necessity of measuring signal residuals over many pixels, as well as identifying the time scales at which control systems must run in order to address both piston and higher spatial frequency drifts. In the case of ideal optics (no surface errors and no dispersion), controlling piston alone to achieve zero optical path difference would suppress the entire PSF in the interferometric dark output, moving all on-axis source light from a point source into the interferometric bright output. Higher spatial frequency geometric and coating errors in the optics introduce scatter that necessitates wavefront correction with deformable mirrors targeting a limited region of effectiveness in the focal plane.

The VNC's control algorithm targets a nearly $60^{\circ}$ wedged region of interest that extends radially from 1.5 and $4.5 \lambda / D$ for a total of approximately 1000 pixels spanning multiple speckles. Averaging over the region reduces measured detector noise by $\mathrm{n}^{1 / 2}$. Variations in the total or average intensity measured over the full detector 
region of interest may be fed back to the delay stage to stabilize the piston error, which can be sensed with $0.5 \mathrm{~nm}$ precision. Transverse speckle motion is associated with all spatial frequencies greater than pure piston that can be suppressed by the deformable mirror, which is controlled using "mode" patterns that may be ordered according to their influence in in the targeted dark zone.

For the data sets presented in Fig. 8, one of the boxes is located near $\sim 2 \lambda / D$ and has an incoherent peak intensity of 3000 counts. The largest single pixel long period intensity drifts in this region are shown at the $\sim 300$ to 600 count level, which corresponds to $\lambda / 20$ to $\lambda / 10$ wavefront errors. The equivalent delay line positional error in the VNC would be about 15 to $30 \mathrm{~nm}$. Introducing $1 \mathrm{~nm}$ controlled perturbations of the DM in a pure piston mode, inserted into the greater control mode scheme at a frequency no lower than $2 \mathrm{~Hz}$ has been tested with promising results. Introducing a fringe tracking control layer to drive the delay line at higher frequencies should prove to be more efficient overall, and it would eliminate the risk of exceeding the range of linear operation.

In practice, for both DM and delay control layers, a control step must be large enough such that the resulting measured signal derivative has a high chance of directing a next step in the right direction. When approaching a fringe minimum inflection point, some step size must be chosen in order to reliably indicate the presence of the inflection. The required step size is a function of the noise properties as well as the instantaneous contrast. To the extent that the noise can be decreased by integration, a combination of both appropriate step size and integration time must be chosen. The integration time must also be small enough such that temporal stability timescales do not compromise the measurement. Ultimately, the mode amplitude to detector signal response can be measured in the control loop, and we aim to automate the system such that control amplitude and detector exposure time adjustments are automated.

Convection due to detectors heating the air inside the chamber is suspected as being the greatest contributor to observed fluctuations, and to a lesser extent, acoustic and mechanical motions, as well as source brightness fluctuations. Running the system under a low pressure condition and removing the optical table isolation are two conditions under which data will be collected once the control-loop operation is running routinely and the limit to in-air performance has been established with high confidence.

\section{END-TO-END JITTER MEASUREMENTS AND ANALYSIS}

First light through the SAINT source to VNC dark detector collected on May 1, 2018 is presented in Fig. 9. At present, this path traverses 36 optical elements and 51 Fresnel interfaces when accounting for only a single arm of the nuller, and 43 elements and 64 interfaces for both the delay stage and deformable mirror (DM) arms.

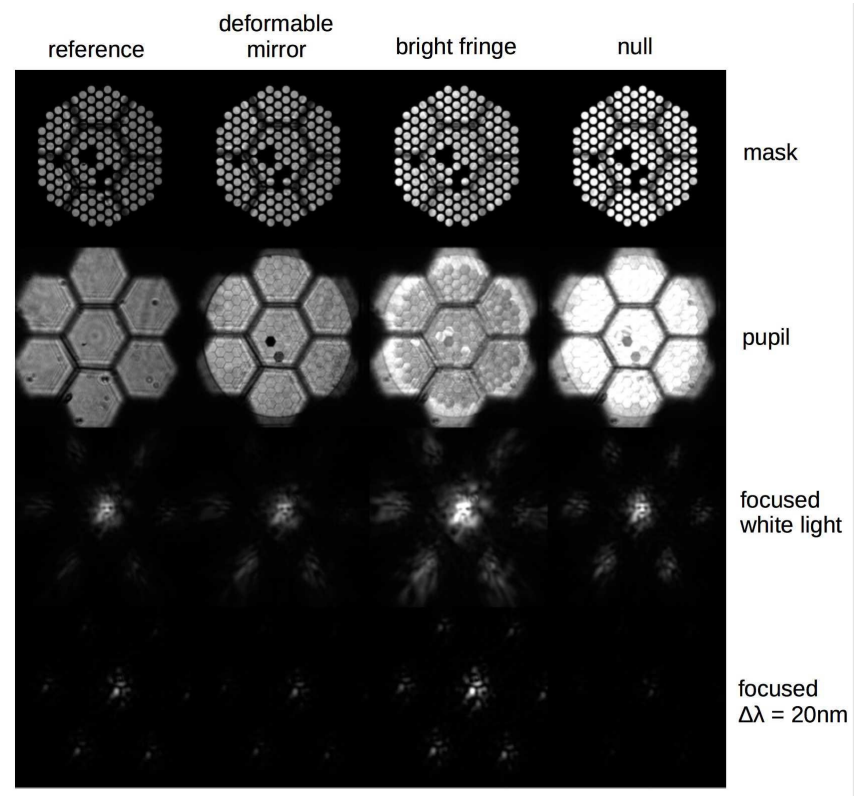

Figure 9. A matrix of imaging conditions through SAINT demonstrating basic end-to-end functionality. Telescope to deformable mirror final magnification and pupil reimaging remains to optimized. A new Lyot mask will also be installed for SAINT once the intermediate MS 1.5 and 2.5 demonstrations have been completed. Columns from left to right show alternating closing shutters to allow light to pass through the reference (delay), then deformable mirror (DM) arms of the VNC, then both shutters open with the delay set locations near bright, then null fringes as observed simultaneously and antisymmetrically on the dark output. The top two rows show the interferometric bright output pupil used for sensing feedback to the DM with (top, labeled "mask") and without the digital mask (below, labeled "pupil") applied that corresponds to the Lyot mask used to block out DM segmented gaps. The bright output observes white light at all times. The bottom half of the matrix shows interferometric dark output focal plane images with unfiltered light above the MS $3 \approx 20 \mathrm{~nm}$ bandpass frames. 
Options exist for reducing the number of elements and interfaces through, e.g., optical contracting and merging fold mirrors and lenses into custom off-axis mirrors. Fewer optics are traversed to reach the other detectors in the system, including the nuller bright output pupil WFS. The top of Fig. 9 shows typical frames recorded by the WFS, which is unfiltered to achieve the minimum possible coherence length and maximize sensitivity to optical path errors near the central fringe.

Representative image quality and stability for the end-to-end optical system are shown in Fig. 10. It is noted that additional work is required to reach the desired telescope to deformable mirror magnification, and a concentrated effort to achieve this goal and align the M1 is presently underway. Similarly, a parallel concentrated effort is underway to closed the loop on the FPS and measure its performance. While these necessary SAINT subsystems have not yet been fully demonstrated, basic computer controls have been established, and basic functionality of the end to end system has been observed down to conservation of energy in the interferometric bright and dark outputs in both unfiltered ("white light") and 20nm bandpass measurements.
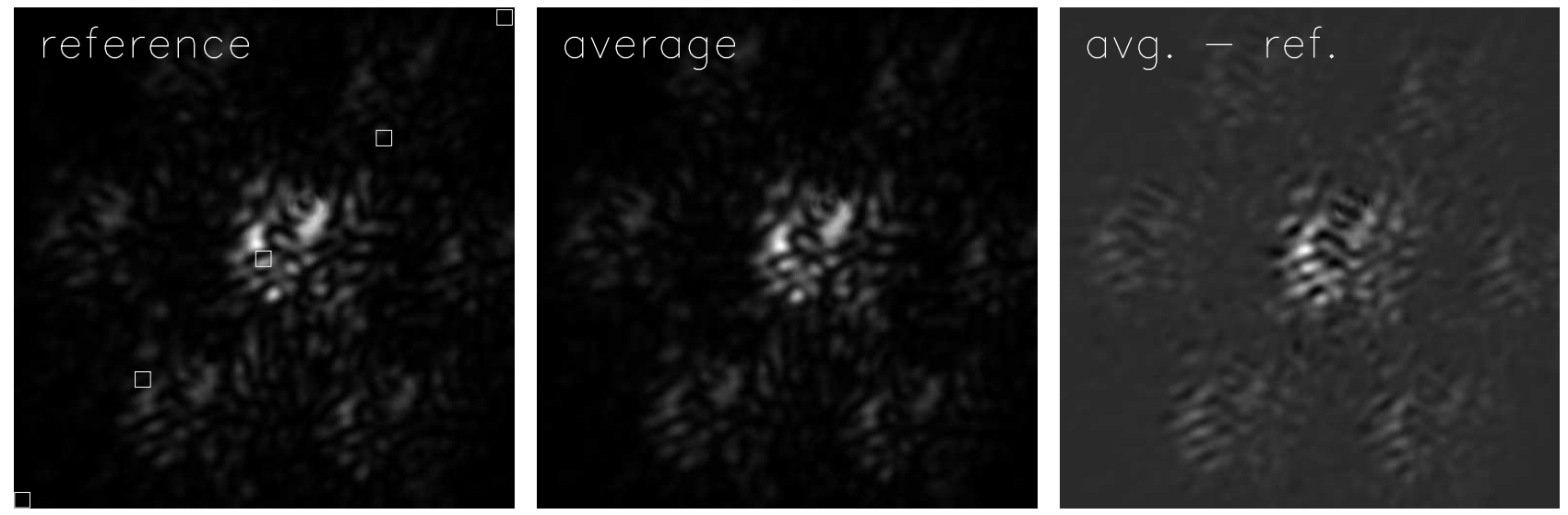

Figure 10. Fundamental image properties for the data set presented in this work. The plate scale is the same as what is presented in Fig. 4, but the image quality is much poorer due to the primary mirror being only coarsely aligned with microns of errors between segments. While the two sets of aperture spread function sidelobes expected for the 1-ring primary and 7-ring DM are not visible, the core and outer sidelobes centroid can be approximately located.

The SAINT end-to-end full frame statistics times series and power spectra are presented in Fig. 11. The backgound and mean in these data are significantly higher than the comparable VNC single arm plots in Fig. 6. The frames were allowed to saturate slightly to maximize the visibility of this starting point PSF viewed in 10 for comparison Fig. 4 at the same linear scale. It is noted that the time series and power spectra for the image data moments are correlated, which is attributed to interferometric path error fluctuations and not source brightness or PSF shape fluctuations. Beyond these marks, given the preliminary nature of these plots, further analysis is left to future work.
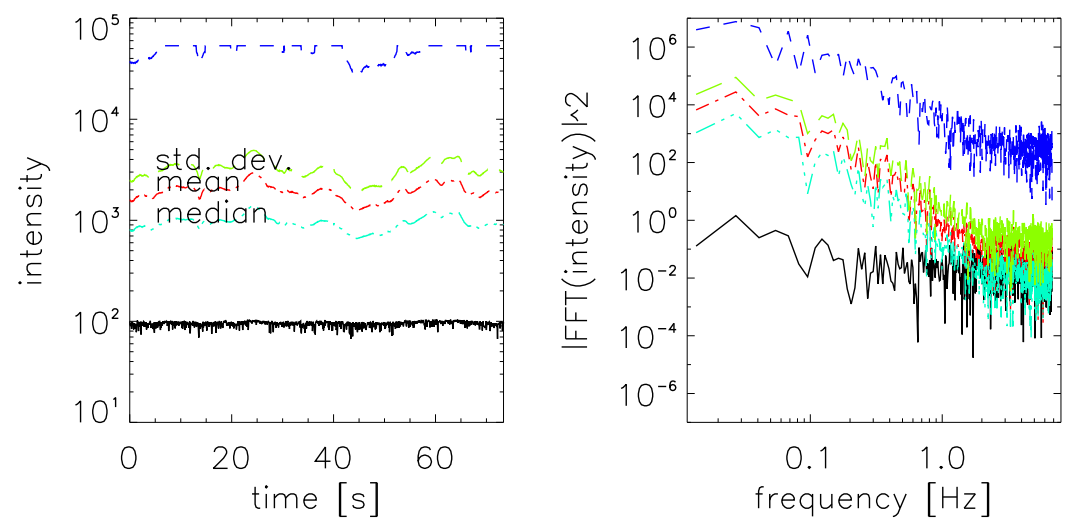

Figure 11. The end-to-end SAINT version of Fig. 5, but with light traversing both arms of the VNC, coherently interfering. The mean and RMS variation of the maximum pixel value over the field of view are 48289 and 7565 ADU, respectively. It is noted that the image was allowed to saturate in order to reveal more of the higher-order PSF structure. The mean and RMS values of the frame mean time series is 1952 and 352 ADU, respectively. 

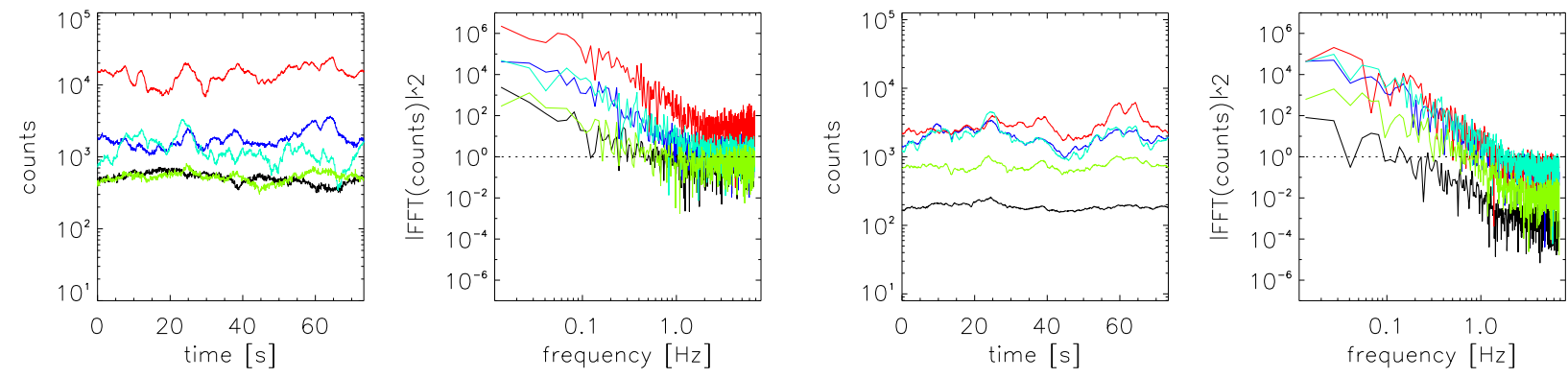

Figure 12. SAINT single pixel (left) and box-averaged (right) time series and power spectra at the locations identified in Fig. 10. Stability should be compred to the that of nulling plots in Fig. 8, not the delay arm only plots shown in Fig. 6.

In comparison to the VNC interferometric plots shown in Fig. 8, Fig. 12 plots SAINT end-to-end interferometric and box average data times series. It is important to again note that fluctuations in intensities are attributed primarily to drifts in the nuller rather than changes in the PSF structure, which, as can be discerned from Fig. 10, is relatively stable. Again, as these data are presented as an initial benchmark, further discussion is left to future work.

Finally, we present in brief the significant pointing jitter observed through the full SAINT end-to-end system, which is plotted in Fig. 13. This measurement represents a significant departure from how the instrument is intended to operate in practice since the fast and fine pointing sensitive ATC is to be used to sense light coming directly from the telescope before being injected into the VNC. Nonetheless, the measurement provides a useful baseline to compare to FPS performance when it becomes operational. It should be noted that these measurements were performed with the telescope being only partially enclosed. The components used in the system have been selected to stabilize pointing to exceed levels observed, presented, and discussed in Fig. 7 in Sec. 3 .
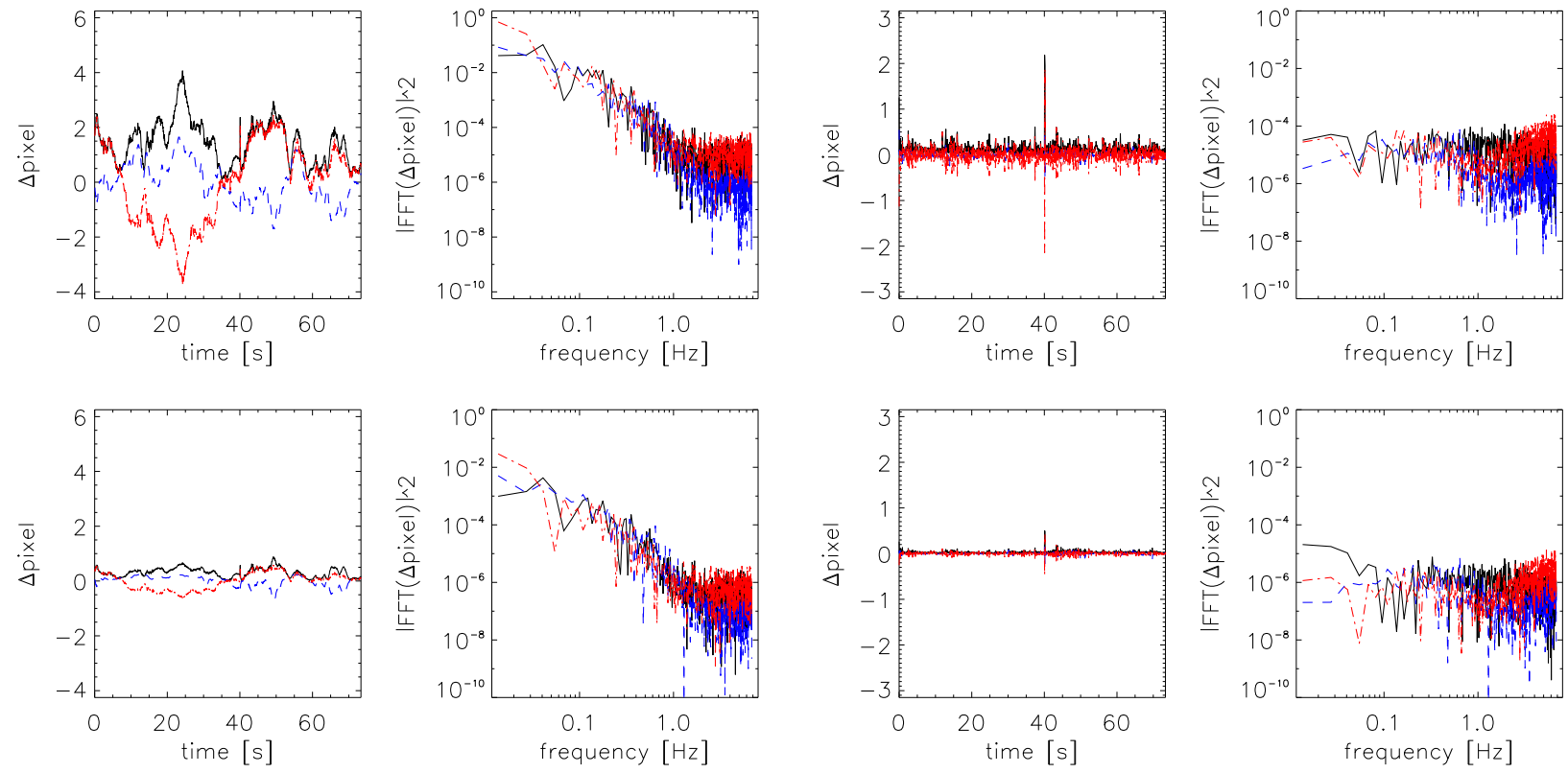

Figure 13. Similar to Fig. 7, the SAINT end-to-end interferometric centroid jitter time series and power spectra plotted for both $\mathrm{x}$ and y directions, as well as magnitude relative to the mean centroid location (top), and previous frame "instantaneous" centroid drift (bottom). The right plots simulate the residual error following correction with the SAINT FPS by saving frames shifted by fractional pixels determined from the first round of centroid calculations, then recalculating centroids for the shifted sets. 


\section{SUMMARY}

We have presented filtered diffraction and stability measurements of the VNC and SAINT. Diffraction enables instantaneous contrasts at demonstration-critical off-axis angles on the order of 1-2 orders of magnitude, while the detector dynamic range and noise gives four orders of magnitude elsewhere within the targeted dark region of the high-contrast focal plane. Delay line optical path error stabilization to be achieved at the 1nm RMS or better level in the VNC should be possible by monitoring full frame statistics, and with comparable and better deformable mirror control, the additional six orders of magnitude and better contrast beyond what is provide by diffraction should be attainable through continuous closed-loop control WFS/C.

SAINT aims to advance coronagraphic techniques for future segmented aperture telescopes. SAINT integrates the VNC with an actively-controlled hexagonal array segmented mirror telescope via a FPS. A successful SAINT effort will culminate in a demonstration of high-contrast imaging with a complex aperture enabled by sequential wavefront control. This SAT/TDEM-funded work specifically targets achieving a contrast of $10^{-8}$ over a $1 \lambda / D$ diameter circular area centered at $4 \lambda / D$ using a filtered source spectral bandpass of $20 \mathrm{~nm}$ centered on $633 \mathrm{~nm}$. A stretch goal will be to achieve $10^{-9}$ contrast at a smaller IWA, possibly $\sim 3 \lambda / D$, and a broader bandpass of $40 \mathrm{~nm}$. Achieving this will further advance coronagraphy towards being adaptable to segmented aperture telescopes. Prior to attempting to reach the end-to-end MS 3 goal, narrowband performance will need to be repeated as MS 1.5, and broadband high-contrast performance demonstrated for the first time as MS 2.5 with the VNC operating inside its vacuum chamber, independent of the air-side actively-controlled segmented telescope optics. ${ }^{11}$

The Milestones are taken to represent feasible objectives based on current VNC Technology Readiness Levels (TRLs), and the TRL of component technologies, including wavefront sensing and control (WFS/C) with a segmented aperture telescope, fine pointing system, deformable mirror, and delay stage. The SAINT project takes an important step towards enabling exoplanet coronagraphy for segmented apertures and ultimately for future missions such as a Large UV/Optical/Infrared (LUVOIR) telescope ${ }^{12}$ or the Habitable Exoplanet Imager (HabEx). ${ }^{13}$ Upon completion of this effort, sub-system technology will be properly positioned to address the additional steps required to mature this high-contrast imaging approach to TRL 6 . The majority of the requisite hardware and software integration has been completed and tested, and the demonstration phase has begun, and final results are expected to be forthcoming.

\section{ACKNOWLEDGMENTS}

The authors acknowledge support from the NASA/GSFC Internal Research and Development (IRAD) and NASA Strategic Astrophysics Technology (SAT) Technology Development for Exoplanet Missions (TDEM) programs.

\section{REFERENCES}

1. B. P. Crill and N. Siegler, "Space technology for directly imaging and characterizing exo-Earths," Proc. SPIE 10398, p. 103980H, Sept. 2017.

2. B. A. Hicks, R. G. Lyon, P. Petrone, M. Ballard, M. R. Bolcar, J. Bolognese, M. Clampin, P. Dogoda, D. Dworzanski, M. A. Helmbrecht, C. Koca, and R. Shiri, "The Segmented Aperture Interferometric Nulling Testbed (SAINT) I: overview and air-side system description," Proc. SPIE 9904, p. 990420, July 2016.

3. B. A. Hicks, M. R. Bolcar, M. A. Helmbrecht, P. Petrone, E. Burke, J. Corsetti, T. Dillon, A. Lea, S. Pellicori, T. Sheets, R. Shiri, J. Agolli, J. DeVries, A. Eberhardt, and T. McCabe, "Segmented Aperture Interferometric Nulling Testbed (SAINT) II: component systems update," Proc. SPIE 10400, p. 104001I, Sept. 2017.

4. R. G. Lyon, M. Clampin, P. Petrone, U. Mallik, T. Madison, and M. R. Bolcar, "High contrast vacuum nuller testbed (VNT) contrast, performance, and null control," Proc. SPIE 8442, Sept. 2012.

5. M. Clampin, R. Lyon, P. Petrone III, U. Mallik, M. Bolcar, T. Madison, and M. Helmbrecht, "Visible nulling coronagraph technology maturation: High contrast imaging and characterization of exoplanets," tech. rep., NASA/Technology Development for Exoplanet Missions Final Report, JPL Document D-80950, https://exep.jpl.nasa.gov/technology/Clampin_Report_FINAL.pdf, 2013. 
6. B. Hicks, R. Lyon, M. Bolcar, M. Clampin, and P. Petrone, "High-contrast visible nulling coronagraph for segmented and arbitrary telescope apertures," Proc. SPIE 9143, 2014.

7. B. A. Hicks, R. G. Lyon, P. Petrone, I. Miller, M. R. Bolcar, M. Clampin, , M. Helmbrecht, and U. Mallik, "Demonstrating broadband billion-to-one contrast with the Visible Nulling Coronagraph," Proc. SPIE 9605, 2015.

8. B. A. Hicks, R. G. Lyon, P. Petrone, I. Miller, M. R. Bolcar, M. Clampin, , M. Helmbrecht, and U. Mallik, "Recent developments with the Visible Nulling Coronagraph," Proc. SPIE 9907, 2016.

9. B. Hicks, M. Bolcar, R. Lyon, M. Clampin, T. Madison, U. Mallik, P. Petrone III, and M. Helmbrecht, "Technology milestone \#2 final report: Achromatic visible nulling coronagraph technology maturation," tech. rep., NASA/Technology Development for Exoplanet Missions, JPL Document D-1547413, https://exoplanets.nasa.gov/exep/technology/TDEM-awards/, 2016.

10. N. Yaitskova, K. Dohlen, and P. Dierickx, "Analytical study of diffraction effects in extremely large segmented telescopes," J. Opt. Soc. Am. A 20, pp. 1563-1575, Aug. 2003.

11. B. Hicks, M. Bolcar, P. Petrone III, and M. Helmbrecht, "Technology milestone \#3 whitepaper: Nulling of an actively-controlled segmented aperture telescope," tech. rep., NASA/Technology Development for Exoplanet Missions, JPL Document D-1549543, https://exoplanets.nasa.gov/exep/technology/TDEM-awards/, 2016.

12. M. R. Bolcar, S. Aloezos, V. T. Bly, C. Collins, J. Crooke, C. D. Dressing, L. Fantano, L. D. Feinberg, K. France, G. Gochar, Q. Gong, J. E. Hylan, A. Jones, I. Linares, M. Postman, L. Pueyo, A. Roberge, L. Sacks, S. Tompkins, and G. West, "The Large UV/Optical/Infrared Surveyor (LUVOIR): Decadal Mission concept design update," 10398, p. 1039809, Sept. 2017.

13. B. Mennesson, S. Gaudi, S. Seager, K. Cahoy, S. Domagal-Goldman, L. Feinberg, O. Guyon, J. Kasdin, C. Marois, D. Mawet, M. Tamura, D. Mouillet, T. Prusti, A. Quirrenbach, T. Robinson, L. Rogers, P. Scowen, R. Somerville, K. Stapelfeldt, D. Stern, M. Still, M. Turnbull, J. Booth, A. Kiessling, G. Kuan, and K. Warfield, "The Habitable Exoplanet (HabEx) Imaging Mission: preliminary science drivers and technical requirements," Proc. SPIE 9904, p. 99040L, July 2016. 